\title{
UN OUTIL D'AIDE Á LA GESTION DE L'ANGUILLE : LE TABLEAU DE BORD ANGUILLLE DU BASSIN LOIRE
}

\author{
A. BAISEZ (1), P. LAFFAILLE (2)
}

(1) LOGRAMI, Université de Rennes 1, Bât. 25, Équipe de Recherche Technologique 52 "Biodiversité Fonctionnelle et Gestion des Territoires", 35042 Rennes. Tél. : 33 (0)2 23236939 - Fax : 33 (0)2 23235138.

E-mail : tableau-anguille-loire@hotmail.fr www.anguille-loire.com

(2) Université de Rennes 1, Bât. 25 Équipe de Recherche Technologique 52 « Biodiversité Fonctionnelle et Gestion des Territoires ", 35042 Rennes.

et Fish-Pass, 8, allée de Guerlédan, 35135 Chantepie. Tél : 33 (0)2 99773211 - Fax : 33 (0)2 99773196.

E-mail : plaffaille@hotmail.com

Reçu le 2 septembre 2004

Accepté le 27 mai 2005

Received September 2, 2004 Accepted May 27, 2005

\section{RÉSUMÉ}

L'anguille européenne (Anguilla anguilla, L.) est longtemps apparue comme une espèce commune, représentant une composante majeure des milieux littoraux et des eaux continentales. La prise de conscience de la diminution de son abondance est réelle et engendre l'émergence de processus de gestion. Ce constat situe l'enjeu du Tableau de Bord Anguille du Bassin Loire mis en place en avril 2002 sous l'égide du COmité de GEstion des POissons Mlgrateurs du Bassin de la Loire, des Côtiers Vendéens et de la Sèvre Niortaise. Son objectif in fine est de contribuer à une gestion et à un développement durable de l'espèce et de ses habitats. II vise à surveiller les caractéristiques des stocks locaux présents et des habitats disponibles au moyen d'une collecte régulière et objective de données synthétisées sous forme d'indicateurs de population et de milieu. Le concept, le fonctionnement actuel et les applications de ce tableau de bord sont ici présentés.

Mots-clés : Tableau de bord, bassin versant, Loire, gestion, anguille.

\section{THE EEL REPORT CARD OF THE LOIRE CATCHMENTS}

\section{ABSTRACT}

Since a long time, the European eel, (Anguilla anguilla, L.), is appeared as a common species and a major fish component in littoral (lagoons, estuaries and coastal marshes) and continental waters (catchments areas, ponds). The significative diminution of its continental abundance has been revealed by many scientific studies. This consciousness is appearing too either among the technical teams working within the different catchments either among the privileged witnesses that are fishermen. It comes to management actions performed at various spatial scales. This report places the context of the Eel Report Card of the Loire catchment, defined in 2000 under the shield of the COMMITTEE of MANAGEMENT MIGRATORY FISH of the Basin of Loire, Vendeans Coasters and Sèvre Niortaise. It has been taking place in April 2002. Its objective in fine is to contribute to a 
management and a sustainable development of the species and habitats. This Eel Report Card organizes a regular and objective acquisition of data characterizing the evolution of the stock in place and of the available habitats. This project brings indicators permitting to fix alarm levels and to value impacts of various measures of management. To lead to well the Eel report Card, it is necessary to end at two indicators of state (quantitative and qualitative): (1) of the eels population to each phase of his biological cycle; (2) of the habitat accessibility for the species within the Loire catchment area. The concept, the working and applications of this Eel Report card are presented in this paper.

Key-words: Report card, catchment area, Loire, management, European eel.

\section{QU'EST CE QU'UN TABLEAU DE BORD?}

Les tableaux de bord sont depuis longtemps utilisés par les économistes et les financiers (voir par exemple, Organisation de coopération et de développement économiques, Centre québécois de développement durable, etc.). Concernant la gestion de l'environnement, quelques tableaux de bord sont menés, essentiellement dans l'optique de la gestion d'un écosystème dans son ensemble (par exemple le tableau de bord du Schéma Directeur pour l'Aménagement et la Gestion de l'Eau dans le bassin Adour-Garonne). Selon I'Union Européenne (EUROPA, 2003), un tel outil doit constituer une source d'information transparente et aisément accessible renseignant sur la situation générale en matière d'aides aux États dans l'Union européenne et sur les activités actuelles de la Commission dans le domaine du contrôle des aides aux États. Cette définition est reprise dans le concept de Tableau de Bord des SDAGEs et SAGEs. II peut être aussi utilisé dans l'optique de la gestion d'une espèce exploitée. Les exemples sont par contre rares et concernent essentiellement des espèces marines ; comme par exemple le charbonnier commun dans le Pacifique Nord (KING et al., 2001).

Concernant la majorité des poissons migrateurs amphihalins, l'enjeu d'un tableau de bord est de contribuer à la fois à la protection de l'espèce et de ses habitats, et au maintien d'une exploitation durable. Cet objectif est ambitieux car leurs cycles de vie complexes se déroulent sur de nombreuses années, nécessitent une migration entre les eaux douces et les eaux marines et sont soumis à une grande diversité de contraintes anthropiques. De plus, la plupart d'entre elles sont exploitées par une grande diversité d'usagers (professionnels, amateurs, braconniers) répondant à une importante demande locale et même internationale, surtout pour l'anguille européenne (Anguilla anguilla L.).

Outre la collecte régulière de données diversifiées et fiables, un tableau de bord doit synthétiser ces informations sous forme d'indices ou d'indicateurs pertinents. Ces indices doivent être validés par des experts et permettre d'obtenir des renseignements à la fois sur l'état du système analysé (ici les poissons migrateurs) mais aussi sur les relations entre les causes et les effets (voir par exemple l'indice poisson développé par OBERDORFF et al., 2001). Ils doivent aussi pouvoir mesurer, à court et à moyen termes, les actes de gestion. Ce n'est qu'à ces conditions qu'un tableau de bord peut être réellement un outil d'aide à la décision.

Pour les poissons migrateurs, cet outil ne doit pas servir qu'à un seul utilisateur mais à tous les usagers et gestionnaires touchant de près ou de loin à cette ressource. Ainsi, ce ne peut pas être exclusivement le Comité de Gestion des Poissons Migrateurs (COGEPOMI) qui l'utilise même si cette structure concentre la plus grande partie des personnes concernées. Ceci serait contraire à la recherche de consensus dans le règlement des conflits entre usages et ressources (voir par exemple FONTENELLE, 2001). En effet, nous pouvons protéger un milieu par tout le panel de protections existantes (zones Ramsar, Znief, Natura 2000, etc.) ou inscrire les espèces dans les différentes annexes (annexes II et V de la Directive habitats-faune-flore, annexe III de la convention de 
Berne, etc.). Mais ces démarches ne seront efficaces qui si les usagers de ces systèmes prennent conscience de l'importance et du bien-fondé d'une gestion concertée même si elle peut déboucher sur des contraintes fortes. C'est par exemple le cas actuellement des zones humides littorales de la baie du Mont-Saint-Michel (LEFEUVRE et al., 2003).

\section{POURQUOI L'ANGUILLE}

En France, les premières démarches sur l'anguille visant à éclairer l'état de l'espèce à l'échelle d'un bassin versant en incluant des données issues des domaines estuariens et dulçaquicoles, des données de pêcheries et des résultats d'inventaires remontent aux années 1980 sur la Vilaine (ELIE et RIGAUD, 1984) et sur la Sèvre Niortaise (GASCUEL, 1987 ; LEGAULT, 1987). Cette démarche indispensable compte tenu des caractéristiques de la phase continentale de croissance de l'anguille a été aussi développée et complétée lors de l'évaluation des voies de migration sur le Bassin du Rhône (FEUNTEUN et al., 2000a; BARRAL, 2000). Les bases du tableau de bord du bassin Loire reposent également sur une telle analyse intégrée des populations d'anguilles à l'échelle d'un bassin versant. Cette approche est également menée sur des sites ateliers tels que les bassins versants du Frémur (LAFFAILLE et al., 2003a), de l'Aulne (BRIAND et al., 2003a) et de la Vilaine (BRIAND, 2003). Sur une autre espèce migratrice, la grande alose, un premier travail d'inventaire des données disponibles ou potentiellement importantes à collecter et des indicateurs synthétiques les plus pertinents à utiliser a été développé sur le territoire COGEPOMI Gironde-Garonne-Dordogne (WOILLEZ et ROCHARD, 2003).

\section{Importance de l'espèce}

Sur le plan ichtyologique, l'anguille européenne (Anguilla anguilla L.) est l'une des espèces occupant la plus grande diversité d'habitats au sein du territoire français (LAFFAILLE et al., 2003b ; 2004a). Elle est en effet susceptible de coloniser tous les types d'habitats accessibles depuis la mer. C'est généralement un élément majeur de nombreux hydrosystèmes fluviaux et estuariens. Par exemple elle représentait, encore récemment, plus de $50 \%$ de la biomasse piscicole des marais atlantiques littoraux (FEUNTEUN et al., 1999) et des systèmes fluviaux du Sud et de l'Ouest de l'Europe où elle est plus particulièrement présente dans les zones les plus en aval, (MORIARTY et DEKKER, 1997 ; données Conseil Supérieur de la Pêche).

Sur le plan écologique, l'anguille représente un excellent «bio-intégrateur » de la qualité de l'environnement (FEUNTEUN, 2002). Sa capacité de bio-intégration peut s'exprimer à différentes échelles d'espace et de temps. Ainsi, lorsqu'elle est abondante dans l'ensemble d'un cours d'eau, cette présence indique l'existence et l'accessibilité d'habitats diversifiés (marais, plaines d'inondation des vallées fluviales, zones apicales des cours d'eau, etc.), mais également une bonne qualité physico-chimique de l'eau ; l'espèce étant particulièrement sensible aux pollutions diffuses (ROBINET et FEUNTEUN, 2002). Cette espèce est aussi un item alimentaire important pour de nombreux prédateurs aquatiques tels que les ardéidés (FEUNTEUN et MARION, 1994). Elle joue aussi un rôle important dans le fonctionnement des hydrosystèmes, plus particulièrement dans les flux de matières (LAFFAILLE et al., 2000). Pour ces raisons, l'anguille européenne doit être considérée comme une espèce parapluie (selon la définition de SIMBERLOFF, 1998), c'est à dire que toute aide à la gestion durable de cette espèce ne peut être que profitable pour tous les autres organismes de la biocénose aquatique et pour le milieu concerné (voir FEUNTEUN, 2002).

Elle représente aussi un intérêt socio-économique important. Tous ses stades continentaux (civelle, anguillette, anguille jaune et anguille argentée) sont exploités pour une production globale annuelle moyenne estimée à environ 20000 tonnes d'anguilles, correspondant à environ 2 billions d'individus, prélevés par an sur toute l'Europe (DEKKER, 
2000a). La civelle est plus particulièrement visée par les européens du Sud, sa production officielle s'établissant entre 500 et 600 tonnes par an. A l'inverse, les européens du Nord exploitent plutôt les anguilles argentées lors de leur migration catadrome. Cette exploitation représente alors environ 6000 tonnes annuelles, soit à peu près 29 millions d'individus (DEKKER, 2000a). En France, cette espèce intéresse environ 2 millions de pêcheurs amateurs dont près de 7000 aux engins. Elle est la principale ressource de 776 pêcheurs professionnels dont 580 en zone fluviale stricte (CHANGEUX, 2002). Selon le Programme PECOSUDE (LÉAUTÉ et CAILL-MILLY, 2003), les pêcheries côtières de civelles représentent en termes économiques, $97 \%$ de la production des marins pêcheurs en zones estuariennes et $62 \%$ de celle des pêcheurs fluviaux. Toutes ces personnes à leur tour, jouent un rôle socio-économique considérable au niveau régional par leur présence dans les zones rurales, les activités qu'ils génèrent (restauration, matériel de pêche, mareyeurs, recherche scientifique, aménageurs, gestionnaires, etc.) et sont aussi des observateurs privilégiés et incontournables de l'environnement.

Sur le plan patrimonial, de très nombreux pêcheurs amateurs aux engins ou aux lignes se focalisent sur l'espèce même seulement pour quelques jours par an. De très nombreuses techniques de pêche existent et sont adaptées tant aux contraintes physiques des milieux exploités que des contraintes juridiques issues de l'histoire sociale, culturelle et économique des espaces concernés. C'est ainsi que certains territoires à forte valeur patrimoniale comme les marais du bassin versant de la Loire (Marais de Brière, Marais Breton Vendéen ou Poitevin pour ne citer que les plus connus) ont longtemps dû le maintien de leur réseau hydrographique à l'exploitation de l'anguille (FEUNTEUN et al., 1992 ; MASSE et RIGAUD, 1998 ; BAISEZ et al., 2000). II a suffi que l'espèce régresse, voire disparaisse faute de gestion appropriée (et de destruction de ses habitats), ou que son exploitation perde de l'intérêt pour des raisons économiques (coût de la main d'œuvre) et surtout juridiques (difficulté d'application des réglementations nationales) pour que cet entretien des réseaux hydrauliques ne soit plus motivé et surtout autofinancé (FEUNTEUN et al., 1992). En conséquence, des milieux comme les marais de Bourgneuf perdent inéluctablement leur intérêt naturel et patrimonial, notamment pour l'anguille, par comblement progressif des espaces aquatiques, faute d'entretien suffisant et de gestion adaptée (LAFFAILLE et al., 2004a).

\section{Constats de diminution}

Son cycle biologique (Figure 1) long et complexe (de 3 à 20 ans environ), sur lequel de nombreuses inconnues existent encore aujourd'hui, et sa répartition sur une vaste aire de croissance compliquent bien sûr le développement d'une gestion durable et coordonnée tant en ce qui concerne son aire de répartition biogéographique qu'à l'échelle des bassins versants. Or, depuis au moins le début des années 1980, l'abondance continentale de l'anguille européenne a décliné à travers toute son aire de répartition (MORIARTY et DEKKER, 1997 ; DEKKER, 2003a). Dans de nombreux pays à travers toute l'Europe de l'Ouest, les immigrations en civelles sont suivies annuellement (DEKKER, 2003a) et permettent de disposer d'un signal sur l'état global de la population. Commencée en 1980, cette régression générale a été observée en continu jusqu'en 1990 où le niveau observé représentait seulement $10 \%$ du niveau initial. Pendant la décennie suivante, les pêcheurs professionnels aux civelles en partie estuarienne de la Loire en ont débarqué dix fois moins qu'à la fin des années 80 (BAISEZ et al., 2002). Ces derrières observations indiquent donc que la régression se poursuit à un rythme régulier. Récemment, certains auteurs affirment même que ce déclin est de $99 \%$ par rapport aux années 60-70 (DEKKER et al., 2003). Toutefois ces derniers chiffres sont sujets à débat. Ainsi, selon PROUZET (comm. pers.), les dernières études sur l'Adour ne mettent pas en lumière une telle diminution sur 30 ans.

Les mêmes constats peuvent être établis pour les anguilles jaunes et argentées même si cette régression a commencé depuis le milieu des années 1960 au moins dans 


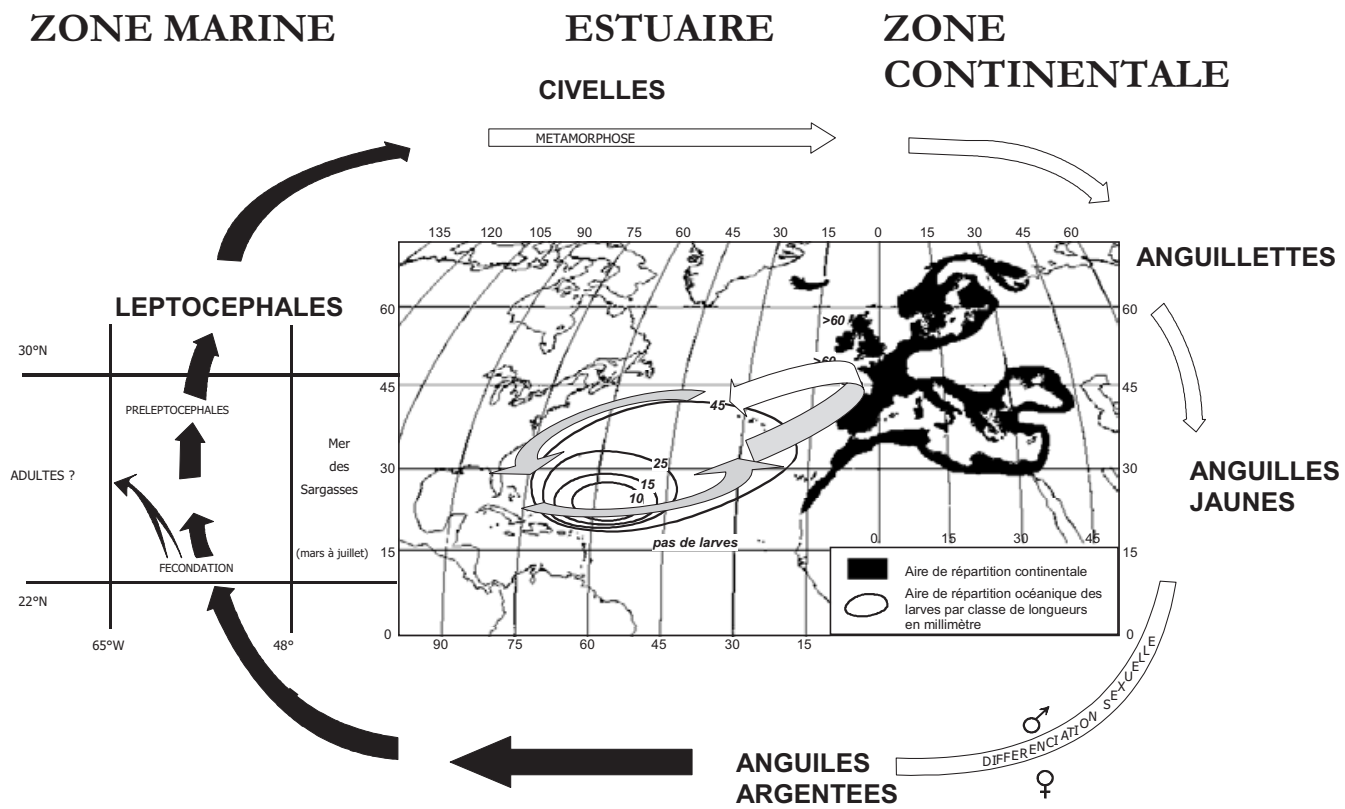

Figure 1

Cycle biologique de l'anguille (Modifié d'après GERMAIN, 1927 ; SCHMIDT, 1922 ELIE et CLEMENT, 1984 ; DEKKER, 2003b).

\section{Figure 1}

European eel life cycle (according to GERMAIN, 1927; SCHMIDT, 1922; ELIE et CLEMENT, 1984; DEKKER, 2003b).

certaines parties de l'aire de répartition (DEKKER, 2003a). Dans la Loire, l'un des bastions de l'espèce, de moins en moins d'individus sont observés dans les zones amont (LAFFAILLE et al., 2004b). Toutefois, l'anguille reste encore abondante dans sa partie centrale de répartition européenne. Sur le Frémur (Bretagne Nord, France), l'une des rivières les plus denses en anguilles jaunes (en moyenne près de 50 individus pour $100 \mathrm{~m}^{2}$ ), les stocks d'anguilles jaunes ne montrent pas d'évolution significative à l'exception des zones les plus en amont où là encore cette espèce à tendance à être moins présente (LAFFAILLE et al., 2005a). De même, lors d'études récentes, MAGALHAES et al. (2002) ont trouvé des anguilles dans $95 \%$ des 166 sites échantillonnées dans le Sud-Ouest de Portugal, où cette espèce reste encore la seconde en terme d'abondance.

Soit par le Groupe National Anguille (ELIE et CLEMENT, 1984 ; RIGAUD, 1997 ; LAMBERT et FEUNTEUN, 1998), soit par divers auteurs (BRUSLE, 1994 ; FEUNTEUN, 2002), ces constats ont été maintes fois répétés au niveau national depuis 20 ans, mais aussi au niveau international (CASTONGUAY et al., 1994; MORIARTY et DEKKER, 1997 ; KIRK, 2003) avec, en parallèle les hypothèses émises pour expliquer ce déclin : causes marines, pollutions, pertes d'habitats, changements climatiques, surexploitation, parasitisme, contaminations chimiques, barrages, etc. (Figure 2). II est certain que ces facteurs doivent agir en synergie. Cependant, les barrages provoquant la déconnexion des milieux aquatiques et entrainant une forte diminution des habitats disponibles ont certainement fortement contribué à ce déclin. Par exemple, MORIARTY et DEKKER (1997) et ICES (2002) ont considéré les effets des barrages sur les habitats disponibles pour les anguilles. La perte moyenne est de l'ordre de $25 \%$ avec des pics à plus de $90 \%$ dans la Péninsule Ibérique où les arrivées potentielles de civelles sont maximales. De ce fait, l'anguille européenne a disparu dans plus de $80 \%$ des bassins versants de la Péninsule 


\section{ZONE MARINE ESTUAIRE ZONE CONTINENTALE}

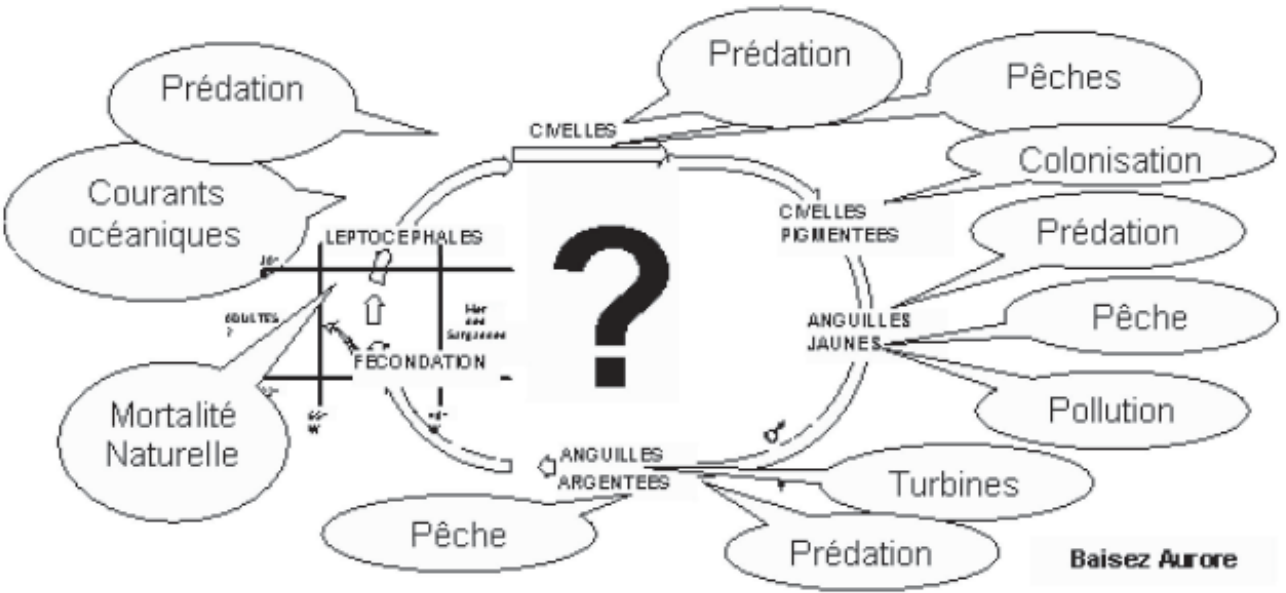

Figure 2

Causes probables de régression de l'espèce.

Figure 2

Probable causes of European eel decrease.

Ibérique, bien qu'elle reste abondante dans les parties non déconnectées de la mer (LOBON-CERVIA, 1999).

\section{Plan de gestion de l'espèce}

Face à ce déclin généralisé, l'Union Européenne, au vu des conclusions du Groupe de travail mixte, Conseil International pour l'Exploration de la Mer (CIEM) et de la Commission Européenne Consultative pour les Pêches dans les Eaux Intérieures (CECPI) de la Food and Agriculture Organisation (FAO), a tiré la sonnette d'alarme en recommandant aux états membres de prendre toutes les mesures en faveur de la restauration de l'espèce. Pour la première fois en 1998, l'Advisory Committee on Fishery Management (ACFM), bras politique du CIEM, considérait que cette espèce était " en dehors de ces limites biologiques de sécurité » et recommandait en particulier " que toutes les pêcheries soient réduites à leur plus bas niveau possible ", " jusqu'à ce qu'un plan de gestion de l'espèce soit mis en place à l'échelle de chaque unité de gestion ". Si le rapport stipule "le plus bas niveau possible " c'est bien pour ouvrir des négociations et par conséquent mettre en place des outils de gestion adaptés. En 2002, l'ACFM recommandait qu'« un plan de restauration du stock d'anguille soit élaboré et mis en œuvre dans la plus grande urgence et que la pêche et toutes les autres mortalités anthropiques soient réduites au plus bas niveau possible jusqu'à ce qu'un tel plan soit accepté et mis en œuvre ". De même, il demandait que «le suivi du recrutement, du stock, des pêcheries et de l'échappement de géniteurs potentiels soient au moins maintenus à l'état actuel pendant qu'un plan de restauration, incluant un programme cohérent de suivi et de recherche, soit accepté et mis en œuvre ". La Commission demandait également que « les pays membres fassent un rapport annuel des fluctuations de leurs populations (d'anguilles) locales et des pêcheries d'anguilles au Working Group ". Pour inciter les pays membres à mettre en place cet outil de gestion, l'ACFM précisait à chaque pays, qu'en l'absence de chiffres, la pêcherie serait considérée comme ayant un impact maximum.

RUSSEL et POTTER (2003) suggèrent alors que le principe de précaution est directement pertinent dans la gestion des stocks d'anguilles européennes. Intégrée dans ce plan global de restauration fixant les grands objectifs à atteindre, l'échelle pertinente 
d'intervention reste bien sûr le bassin versant, car il y est envisageable d'y définir des objectifs concrets de restauration et de gestion de fractions locales de population (FEUNTEUN, 2002). Cette échelle est notamment très souvent la plus pertinente dans la gestion et la conservation des poissons (COLLARES-PEREIRA et COWX, 2004). En outre, de nombreux scientifiques considèrent que chaque fraction de population d'anguilles jaunes abritée au sein d'un bassin versant y subit des aléas particuliers et y révèle une dynamique particulière pouvant être très différente du bassin versant voisin (DEKKER, 2000b). En avril 2002, le groupe anguille du COGEPOMI Loire a donc décidé de mettre en place «le Tableau de Bord Anguille du Bassin Loire » dont l'objectif in fine est de se donner les moyens d'une gestion et d'un développement durable de l'espèce et de ses habitats. En 2003, le COGEPOMI Loire conforta cet outil par une notification au sein du Plan de Gestion des Poissons Migrateurs (Arrêté N²29/DIREN/2003) mentionnant qu'« une des actions prioritaires à mener dans le cadre du plan de gestion 2003-2007 était la mise en place d'un dispositif de suivi de la pêche et des fractions de population d'anguilles du bassin (Loire/Côtiers Vendéens/Sèvre Niortaise) ».

\section{FONCTIONNEMENT DU TABLEAU DE BORD ANGUILLE DU BASSIN LOIRE}

Le Tableau de Bord Anguille du Bassin Loire est dorénavant un outil fonctionnel dans le suivi de l'espèce. C'est un dispositif régulier et objectif d'acquisition des caractéristiques de la fraction de population et du milieu.

\section{Comité de pilotage}

L'élaboration du Tableau de Bord Anguille et le transfert des informations vers les gestionnaires, scientifiques et usagers est sous la responsabilité d'un Comité de pilotage constitué: du maître d'ouvrage LOGRAMI (l'association LOire GRAnds Mlgrateurs) accompagnée de la Direction Régionale de l'ENvironnement Pays de la Loire, de Scientifiques, du Conseil Supérieur de la Pêche, du Syndicat Mixte pour le Développement de l'Aquaculture et de la Pêche, de l'Etablissement Public Loire, de l'Agence de l'Eau Loire-Bretagne et du Conseil Régional des Pays de la Loire. Ce comité valide les documents et les informations du Tableau de Bord. II facilite aussi parfois l'accès aux données pour l'animateur. Enfin, il représente la majorité des acteurs de la gestion de l'espèce et du milieu.

\section{Structure d'accueil indépendante}

Depuis avril 2002, la démarche d'élaboration du Tableau de Bord Anguille du Bassin de la Loire, des Côtiers Vendéens et de la Sèvre Niortaise est effectuée avec la mise en place d'un poste d'animatrice. Cette animation repose sur un financement de l'Etat (via le Plan Loire Grandeur Nature), du Conseil Régional des Pays de la Loire, de l'Agence de l'Eau Loire Bretagne, de I'Etablissement Public Loire et de LOGRAMI. Le Conseil Supérieur de la Pêche apporte son aide technique, scientifique et ses grandes connaissances à la fois du milieu et de l'espèce. L'encadrement scientifique et l'hébergement de l'animatrice sont assurés par l'Université de Rennes 1 (Equipe de Recherche Technologique 52 «Biodiversité fonctionnelle et gestion des territoires »). Leur thématique de recherche très similaire à celle du Tableau de Bord consiste à collecter, organiser et formuler les connaissances écologiques sur la diversité biologique et le fonctionnement des écosystèmes pour être en mesure de fournir les éléments d'aide à la décision nécessaires aux responsables locaux de la gestion des territoires. Ainsi, l'animatrice est accueillie dans une structure lui permettant d'être au maximum indépendante des divers usagers (pêcheurs amateurs, professionnels, etc.) et des gestionnaires (services de l'état, collectivités territoriales, etc.). Cette indépendance est indispensable pour obtenir un maximum d'objectivité et un minimum de pression lors des recommandations aux divers acteurs du Tableau de Bord. 


\section{Types de mission}

Le travail de l'animatrice consiste à organiser la collecte de données auprès des usagers, administrations et gestionnaires. L'ensemble des données centralisées permet une gestion des informations en méta-base (notamment la compilation des données existantes auprès des différents acteurs). La globalisation plus ou moins importante des informations permet de répondre à des interrogations à l'échelle du bassin (30 départements, 9 régions), ce qui correspond à la première échelle de gestion locale, à l'échelle régionale (seconde échelle de gestion) et départementale (troisième échelle de gestion). Mais, cette démarche peut permettre aussi, en amont, de répondre, notamment aux attentes de l'Etat et de l'Europe (par exemple OUEST GRANDS MIGRATEURS, 2003).

Les retombées en termes d'aide à la gestion consistent en l'élaboration d'avis scientifiques sur la biologie de l'espèce et la qualité du milieu et en la mise en place des protocoles de suivis pour les acteurs partenaires (Direction Départementale de l'Agriculture et de la Forêt, Fédération de pêche, Association de protection de la nature, Parc Naturel, etc.). Enfin, Les données validées par le COGEPOMI et le Comité scientifique contribuent au bilan national demandé par les instances européennes via le groupe CIEM/CECPI.

Le recensement des différentes sources d'informations a permis de révéler des carences ou des incertitudes dans les réseaux en place pour aboutir aux indicateurs de population et de milieu (Figure 3). Le repérage de ces lacunes entraîne la mise en place de programmes d'études (Figure 4) coordonnés et complémentaires. Par exemple, l'étude de la qualité des fiches de déclaration des professionnels de l'estuaire de la Loire (CRTS, DPMA, Tableau de Bord Anguille Loire) sert de base à la mise en place du modèle d'estimation du flux de civelles (Ifremer, INDICANG, Tableau de Bord Anguille Loire).

\section{Échelle de travail}

L'échelle de travail correspond aux territoires du COGEPOMI Loire. Le Tableau de Bord Anguille du Bassin Loire comprend donc les zones géographiques de la Loire, de ses affluents, de la Sèvre Niortaise et des Côtiers Vendéens représentant 9 régions et 30 départements couvrant une superficie de $117800 \mathrm{~km}^{2}$ pour la Loire (1/5 de la France)

Indicateurs de Milieu

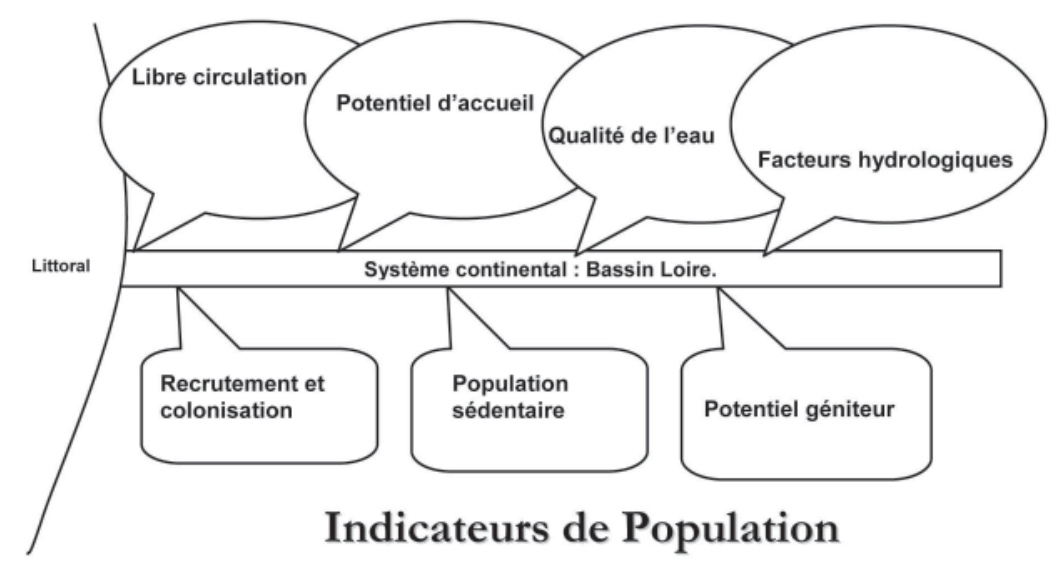

Figure 3

Les indicateurs de milieux et de population.

Figure 3

Habitat and population indicators. 


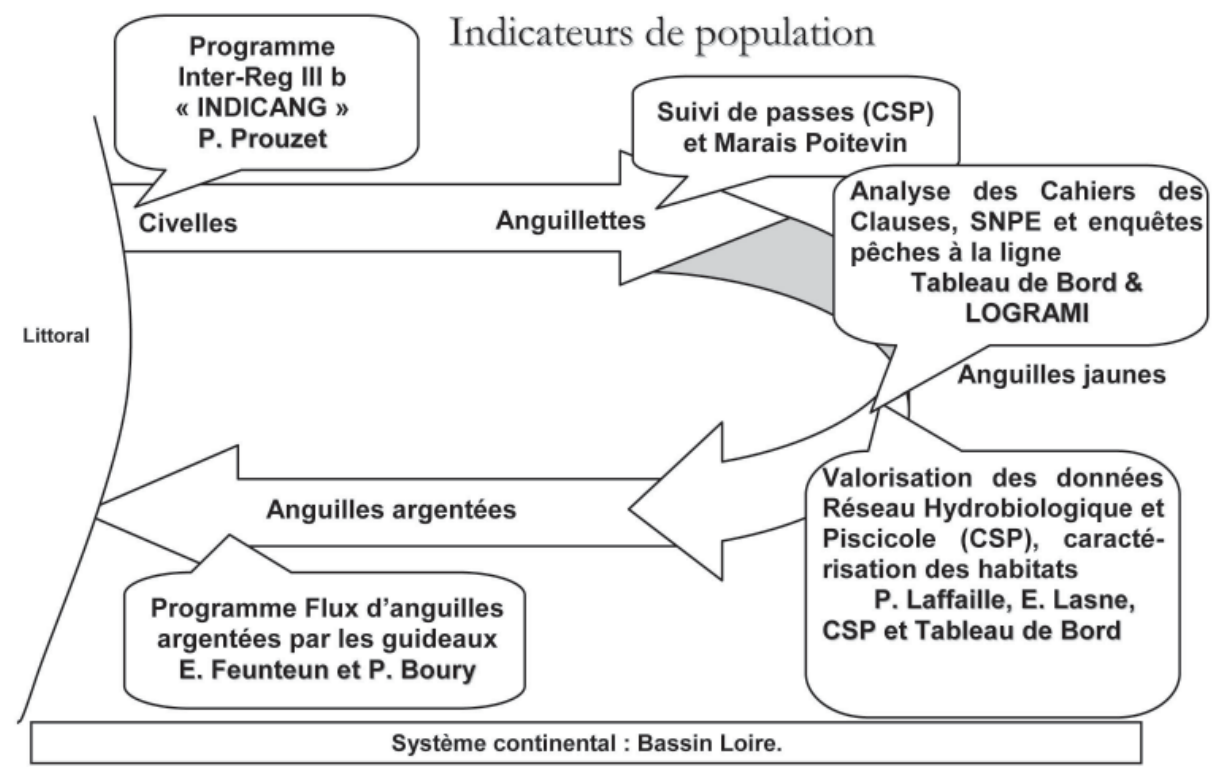

Figure 4

Liste des études complémentaires mises en œuvre.

Figure 4

List of the performed supplementary studies.

et $15000 \mathrm{~km}^{2}$ pour les bassins versants supplémentaires. La gestion concrète de l'espèce et de ses habitats, menée au niveau du bassin versant, doit venir s'intégrer à une démarche globale prenant en compte l'ensemble de l'aire de répartition. C'est pourquoi, ces travaux sont intégrés dans une échelle plus vaste (Arc atlantique) avec un souci de standardisation des indicateurs synthétiques retenus dans les différents bassins de cette zone importante pour l'espèce (Programme Européen INDICANG, chef de file P. Prouzet IFREMER).

\section{THÉMATIQUES RETENUES ET DONNÉES RECUEILLIES}

Cet outil doit synthétiser les données collectées au travers d'indicateurs permettant de fixer des niveaux d'alerte et d'évaluer les impacts de mesures de gestion. Pour mener à bien le Tableau de Bord, il est nécessaire d'aboutir in fine à deux groupes d'indicateurs d'état des milieux colonisables par l'espèce et de la fraction de la population d'anguilles présente dans le bassin à chaque phase de son cycle biologique. Ces indicateurs devant être déclinés en termes quantitatifs et qualitatifs.

\section{Les descripteurs de milieu retenus}

\section{La libre circulation}

S'appuyant sur une expertise de libre circulation développée par le Conseil Supérieur de la Pêche au niveau de chaque obstacle du bassin, l'indice d'accessibilité du réseau hydrographique permet de visualiser les points de blocage à la montaison des anguilles et les séries d'obstacles pouvant alors être à l'origine des faibles densités observées. De même cet indicateur de libre circulation doit intégrer la nécessaire transparence des obstacles lors de la phase de dévalaison des anguilles argentées (STEINBACH, 2003). Les obstacles sont repérés sur les axes du réseau hydrographique. En cas d'aménagement complexe, l'ensemble des ouvrages associés à un même étage hydraulique (bief au sens large) fait l'objet d'une analyse globale aboutissant à un seul résultat. La franchissabilité des ouvrages est alors évaluée 
suivant six classes de franchissabilité, allant de l'obstacle effacé (classe 0) jusqu'au barrage infranchissable (classe 5). Les conditions d'observation et les critères d'appréciations doivent être adaptés à la période (entre mai et fin juillet - LAFFAILLE et al., 2000) et au comportement migratoire particulier de l'espèce (capacité de nage dans les écoulements à faible vitesse et capacité de reptation sur les surfaces mouillées et rugueuses - LEGAULT, 1988). Les facteurs pris en compte sont alors : (1) la hauteur de chute, (2) la verticalité ou l'inclinaison des parements aval, (3) le lissage ou la rugosité des surfaces d'écoulement et d'appui, (4) la verticalité ou l'inclinaison latérale des zones de transition avec la berge et (5) la diversité des voies de franchissement possibles (STEINBACH, 2004).

\section{Nature et importance des habitats disponibles}

Cet indice, définissant le potentiel d'accueil, doit s'appuyer sur les suivis de qualité des milieux, les expertises des cours d'eau et leur évaluation en termes de surface colonisable potentielle et réelle, de valeur et de fonction (alimentation, refuge, passage) vis à vis des différents stades biologiques de l'anguille. Cet indice est important car il est actuellement estimé que près de $33 \%$ des habitats originellement disponibles à cette espèce ne le sont plus pour des raisons naturelles et/ou artificielles (MORIARTY et DEKKER, 1997). Or la qualité et la quantité des habitats disponibles sont un des facteurs les plus importants pouvant expliquer l'état des stocks continentaux d'anguilles (LAFFAILLE et al., 2004c).

\section{Caractéristiques hydrologiques}

Révélant des phénomènes particuliers d'ordre naturel (pluviosité, états de sécheresse, etc.) ou anthropiques (soutien de débits réservés par exemple), ils doivent être pris en compte pour interpréter et relativiser les variations des indicateurs d'état de la population et pour moduler les indices de potentiel d'accueil et de libre circulation. Des facteurs comme la température, le coefficient de marée, le débit, la phase lunaire influencent le flux migratoire des civelles (voir par exemple DE CASAMAJOR et al., 1999 ; JELLYMAN et LAMBERT, 2003) et des anguilles de dévalaison (voir par exemple FEUNTEUN et al., 2000 b ; DURIF et al., 2003). Leur prise en compte doit être intégrée dans la définition des protocoles devant permettre des mesures de flux.

\section{La qualité de l'eau}

Ce paramètre doit permettre d'apprécier en termes de quantité et qualité d'anguilles le potentiel d'accueil que révèle telles ou telles zones du bassin. Sa prise en compte s'appuiera sur le classement des cours d'eau selon leur eutrophisation phytoplanctonique et/ou leur qualité physico-chimique (métaux lourds et pesticides) prochainement rendue obligatoire afin de se conforter à la Directive Cadre Eau. II sera important de vérifier l'adéquation entre les paramètres mesurés, les seuils de détection et les exigences biologiques de l'anguille et les conséquences en termes de potentialités reproductrices des individus (ROBINET et FEUNTEUN, 2002).

\section{Les descripteurs par stade biologique}

La gestion de l'anguille au sein du bassin Loire doit tenir compte de l'ensemble des stades de l'espèce en phase continentale. Nous considérons ainsi les trois phases de développement de l'anguille dans le bassin Loire.

\section{Le recrutement}

Les civelles et les anguillettes correspondent à la phase de recrutement du bassin. La dynamique et l'importance de cette colonisation du milieu continental sont susceptibles d'être mesurées par trois indices. Tout d'abord, l'analyse des stades et des flux de civelles en migration portée (GASCUEL, 1986), très en aval dans l'estuaire, peut renseigner sur 
le niveau du recrutement estuarien en identifiant les nouveaux individus entrant dans le système. Ce signal témoigne de l'état de santé global de la population européenne d'anguilles, mais aussi à l'entrée du système (voir par exemple LEFEBVRE et al., 2003). A l'autre extrémité de l'estuaire, l'analyse des civelles en migration nagée (GASCUEL, 1986), en amont de la limite de marée dynamique, fournit une indication du recrutement fluvial (LEGAULT, 1994). Le niveau de cet échappement estuarien résulte du taux d'exploitation exercé par les pêcheurs sur le flux de civelles, du taux de sédentarisation en estuaire et du taux de mortalité naturelle sur ces jeunes stades appliqués entre ces points amont et aval (BRIAND et al., 2003a). Enfin, une partie du recrutement fluvial est assurée par les anguillettes, jeunes anguilles de 8 à $20 \mathrm{~cm}$ ayant passé quelques mois en eau douce (LEGAULT 1994 ; LAFFAILLE et al., 2000). L'ensemble de ces indices peuvent être issus de suivis des pêcheries (BRIAND et al., 2003b) et/ou des passes-pièges à anguilles (LEGAULT, 1994) ainsi que de la mise en œuvre d'opérations spécifiques, manœuvres d'ouvrage (LEGAULT, 1990) ou pêcheries expérimentales (BRIAND et al., 2003a).

\section{Le stock sédentaire}

Les anguilles jaunes représentent la phase sédentaire de croissance. Les caractéristiques de cette phase peuvent être déclinées en termes d'abondances, de structures démographiques, et de proportion des sexes. Leur déclinaison fournit un indice tant qualitatif que quantitatif de la population en place dans les différents compartiments du bassin (voir par exemple FEUNTEUN et al., 1998). Les pêches expérimentales, les suivis des captures des pêcheurs professionnels et amateurs et les inventaires piscicoles adaptés ou non à l'anguille constituent les sources de données pouvant permettre d'atteindre cet objectif (LAFFAILLE et al., 2004b ; LAFFAILLE et al., 2005b).

\section{Le potentiel reproducteur}

Les anguilles argentées représentent le potentiel reproducteur issu du bassin. Nous pouvons en Loire obtenir un indicateur quantitatif et qualitatif de dévalaison par l'observation des captures professionnelles au niveau d'Ancenis, en amont de Nantes (FEUNTEUN et BOURY, 2003). Les suivis effectués permettent ainsi l'estimation de la pression de pêche et de son impact et l'analyse des caractéristiques du flux dévalant en termes de proportion des sexes, de tailles, de repérage des stades d'argenture, de qualité des individus (pourcentage de graisse, maturation des gonades, contamination parasitaire, etc.).

\section{LES DONNÉES}

\section{Origines}

Les données sont souvent issues des réseaux de suivis déjà en place par ailleurs (Conseil Supérieur de la Pêche, Dlrection Régionale de l'ENvironnement, Agence de l'eau Loire Bretagne, etc.). Elles sont transmises au Tableau de Bord après validation des organismes fournisseurs et restent leur entière possession. Ces données sont retransmises aux acteurs sous forme compilée (cartographies, graphiques, analyses statistiques, etc.) en réponse à leurs interrogations sur la thématique Anguille et/ou Milieux associés. D'autres données sont issues de collectivités territoriales, associations ou organismes privés suivant les mêmes obligations d'appartenance. Les démarches de collectes sont rendues pérennes par la mise en place de conventions entre les organismes fournisseurs et le maître d'ouvrage du Tableau de Bord.

\section{Diffusion}

Les informations diffusées sont toujours «digérées» pour une meilleure compréhension et une interprétation plus facile par des acteurs non spécialisés (grand 
public, élus, administrations, etc.). Nous pouvons classifier le flux d'informations issu d'un Tableau de Bord en deux catégories: «Pull » et «Push » (CAN et al., 1999). Le flux «Pull » concerne une information disponible et accessible qui est destinée à « ceux qui savent qu'ils ne savent pas »; la démarche de recherche de l'information est donc volontaire dans ce cas de figure. Cela correspond aux demandes d'avis du COGEPOMI pour l'aide à la prise de décision, mais également à la recherche volontaire de documents téléchargeables sur l'espèce et son milieu sur le site Internet (www.anguille-loire.com). A l'inverse, le flux «Push », plus accrocheur puisque diffusé à l'insu de l'utilisateur, est destiné à " ceux qui ne savent pas qu'ils ne savent pas ", le destinataire va enrichir sa connaissance à la lecture de cette information. Cette démarche a motivé la mise en place d'une lettre d'information «Paroles d'anguilles » qui donne une information semestrielle aux principaux acteurs du bassin Loire. Les journées de colloques «Rencontres Anguilles en Loire » constituent également une restitution du savoir vers les usagers et gestionnaires. Enfin, le flux «Push » s'adresse également à « ceux qui ne savent pas qu'ils savent ", dans ce cas là, l'information revêt un rôle de révélateur pour l'utilisateur. II sert au retour d'information des différents acteurs (scientifiques, pêcheurs, animateurs de SAGE, administrations, etc.) vers le Tableau de Bord.

\section{CONCLUSIONS}

Les tableaux de bord sont de plus en plus employés, mais ils sont très divers selon les objectifs qu'on leur assigne. Concernant les populations piscicoles migratrices, chaque indicateur doit apporter des informations en termes de gestion du système (pression de capture, connexité du système, qualité du milieu, etc.) mais doit également être à même de participer à l'élaboration d'un modèle global de gestion de la population. Ainsi, la gestion de l'anguille au sein du bassin versant de la Loire doit tenir compte de l'ensemble des stades continentaux de l'espèce (la phase de recrutement, la phase sédentaire et la phase reproductrice). Pour ce faire, le Tableau de Bord utilise toutes les données disponibles sur l'anguille et son habitat auprès des administrations, scientifiques et usagers sur l'ensemble du bassin versant. II est par conséquent à l'interface entre les usagers, les gestionnaires et les scientifiques, et à la disposition de ces trois composantes.

Ce premier travail de recensement des sources d'informations, de collecte des données, de premières restitutions (rapport, site Internet, diaporamas) a pleinement occupé les 2 premières années de fonctionnement de ce tableau de bord. Alors que l'approche Bassin Versant est aujourd'hui reconnue comme indispensable dans bien des domaines ayant trait à la gestion de l'eau, des milieux aquatiques et des ressources associées, il apparaît en effet une grosse difficulté de regrouper les informations disséminées dans les différentes structures administratives ou techniques. Ce premier objectif est en bonne voie d'être atteint, il reste maintenant à avancer dans l'interprétation de ces données, dans leur restitution sous forme de signaux ou d'indicateurs clairement établis et partagés par le plus grand nombre d'acteurs. Cette phase est enclenchée notamment en s'associant à la démarche Indicang développée sur 12 bassins de la Côte atlantique.

Cet exemple concret de surveillance structurée à grande échelle peut très facilement être mis en place dans d'autres écosystèmes et/ou pour d'autres espèces dont la conservation et/ou la restauration devient urgente.

\section{REMERCIEMENTS}

Un grand remerciement à tous les membres du Comité de pilotage du Tableau de Bord Anguille du Bassin Loire, ainsi qu'aux différents financeurs sans qui ce projet n'aurait pu voir le jour : LOGRAMI, la DIREN Pays de la Loire, le Conseil Régional des Pays de la Loire, I'Agence de l'Eau Loire Bretagne, l'Etablissement Public Loire, le Conseil 
Supérieur de la Loire et l'Université de Rennes 1. Nous remercions aussi E. FEUNTEUN, C. RIGAUD, P. PROUZET pour leurs remarques et suggestions constructives. Pour plus de renseignements nous vous conseillons de visiter le site www.anguille-loire.com.

\section{BIBLIOGRAPHIE}

BAISEZ A., RIGAUD C., FEUNTEUN E., 2000. Hétérogénéité de répartition de l'anguille européenne (Anguilla anguilla, L.) observée par pêche électrique dans un marais endigué de la côte atlantique française (Marais breton). Cybium, 24, 23-32.

BAISEZ A. et collaborateurs, 2002. Rencontres scientifiques, l'anguille en Loire. Rapport Tableau de Bord Anguille du Bassin Loire, 50 p.

BARRAL M., 2000. Etude préliminaire à la mise en place d'un Tableau de bord Anguille sur le bassin Rhône Méditerranée Corse. Rapport Association Migrateurs Rhône Méditerranée, $93 \mathrm{p}$.

BRIAND C., 2003. La gestion de l'anguille sur le bassin versant de la Vilaine. Colloque «les poissons migrateurs, un patrimoine régional ». Rennes, le 16 octobre 2003.

BRIAND C., FATIN D., LAFAGE D., LAFFAILLE P., 2003a. Gestion du stock d'anguille du bassin versant de l'Aulne. Rapport Comité Local des Pêches Nord Finistère, Fédération du Finistère de Pêche et de Protection de Milieux Aquatiques, Institut d'Aménagement de la Vilaine, Université de Rennes 1, Fish-Pass, $20 \mathrm{p}$.

BRIAND C., FATIN D., FONTENELLE G., FEUNTEUN E., 2003b. Estuarine and fluvial recruitment of the European glass eel, Anguilla anguilla, in an exploited Atlantic estuary. Fisheries Management and Ecology, 10, 377-384.

BRUSLE J., 1994. L'anguille européenne Anguilla anguilla, un poisson sensible aux stress environnementaux et vulnérable à diverses atteintes pathogènes. Bull. Fr. Pêche Piscic., 335, 237-260.

CAN D.C., FOLLIARD K.A., HUANG S.T., KENNY A.M., McGREGOR J.A., 1999. How to implement a successful communication program: a case study. Beel Labs Technical Journal, 172-180.

CASTONGUAY M., HODSON P.V., MORIARTY C., DRINKWATER K.F., JESSOP B.M., 1994. Is there a role of ocean environment in American and European eel decline? Fisheries Oceanography, 3, 197-203.

CHANGEUX T., 2002. L'effort de pêche ciblant l'anguille dans les eaux libres françaises en 2000. Rapport Conseil Supérieur de la Pêche, 8 p.

COLLARES-PEREIRA M.J., COWX I.G., 2004. The role catchment scale environmental management in freshwater fish conservation. Fisheries Management and Ecology, $11,303-312$.

De CASAMAJOR M.N., BRU N., PROUZET P., 1999. Influence de la luminosité nocturne et de la turbidité sur le comportement vertical de migration de la civelle d'anguille (Anguilla anguilla L.) dans l'estuaire de l'Adour. Bull. Fr. Pêche Piscic., 355, $327-$ 347.

DEKKER W., 2000a. A Procrustean assessment of the European eel stock. ICES Journal of Marine Science, 57,938-947.

DEKKER W., 2000b. The fractal geometry of the European eel stocks. ICES Journal of Marine Science, 57, 109-121.

DEKKER W., 2003a. Status of the European eel stock and fisheries. In: AIDA K., TSUKAMOTO K., YAMAUCHI K., Eel biology, eds Springer-Verlag Tokyo. 
DEKKER W., 2003b. On the distribution of the European eel (Anguilla anguilla) and its fisheries. Canadian Journal of Fisheries and Aquatic Sciences, 60, 787-799.

DEKKER W., CASSELMAN J.M., CAIRNS D.K., TSUKAMOTO K., JELLYMAN D., LICKERS H., 2003. Worldwide decline of eel recources necessitates immediate action. Fisheries, 28, 28-30.

DURIF C., ELIE P., DUFOUR S., MARCHELIDON J., VIDAL B., 2000. Analyse des paramètres morphologiques et physiologiques lors de la préparation à la migration de dévalaison chez l'anguilla européenne (Anguilla anguilla) du lac de Grand-Lieu (Loire-Atlantique). Cybium, 24, 63-74.

ELIE P., CLEMENT O., 1984. Rapport de synthèse du Groupe national Anguille (La Rochelle, 1983), $60 \mathrm{p}$.

ELIE P., RIGAUD C., 1984. Etude de la population d'anguille (A. anguilla) de l'estuaire et du bassin versant de la Vilaine : pêche, biologie, écologie. Examen particulier de l'impact du barrage d'Arzal sur la migration anadrome. Université Rennes, Cemagref Bordeaux, URFDAAPP, $175 \mathrm{p}$.

EUROPA 2003 http://europa.eu.int/index_fr.htm

FEUNTEUN E., 2002. Restoration and management of the European eel: an impossible bargain? Ecological Engineer, 18, 575-591.

FEUNTEUN E., RIGAUD C., ELIE P., 1992. Le marais doux endigué de BourgneufMachecoul : premiers éléments de connaissance du peuplement piscicole, relation ichthyofaune habitat, mise en évidence des problèmes majeurs de gestion. Revue des Sciences de l'Eau, 5, 509-527.

FEUNTEUN E., MARION L., 1994. Impact of heron (Ardea cinerea) on fish communities: the case study of the main European colony. Hydrobiologia, 279/280, 327-344.

FEUNTEUN E., ACOU A., GUILLOUËT J., LAFFAILLE P., LEGAULT A., 1998. Spatial distribution of an eel population (Anguilla anguilla L.) in a small coastal catchment of Northern Brittany (France). Consequences of Hydraulic works. Bull. Fr. Pêche Piscic., 349, 129-139.

FEUNTEUN E., RIGAUD C., ELIE P., LEFEUVRE J.-C., 1999. Les peuplements piscicoles des marais littoraux endigués atlantiques: un patrimoine à gérer? Le cas du marais de Bourgneuf Machecoul (Loire-Atlantique, France). Bull. Fr. Pêche Piscic., 352, 63-79.

FEUNTEUN E., BOULIER J., BRIAUDET P.E., LAFFAILLE P., 2000a. L'anguille du Rhône aval : étude préalable à l'élaboration d'un protocole de suivi et de restauration. Rapport COGEPOMI Rhône-Méditerranée-Corse, $107 \mathrm{p}+$ annexes.

FEUNTEUN E., ACOU A., LAFFAILLE P., LEGAULT A., 2000b. European eel (Anguilla anguilla): prediction of spawner escapement from continental population parameters. Canadian Journal of Fisheries and Aquatic Sciences, 57, 1627-1635.

FEUNTEUN E., BOURY P., 2003. Premiers résultats de l'étude d'estimation des flux d'anguilles argentées du basin versant Loire et perspectives. Secondes Rencontres Scientifiques « l'anguille en Loire », 7 mars 2003, Angers.

FONTENELLE G., 2001. Le programme Urbamont (usages et ressources an baie du Mont Saint-Michel) : un essai de convergence disciplinaire sur un site complexe. Natures Sciences Sociétés, 9, 68-70.

GASCUEL D., 1986. Flow-carried and active swimming migration of the glass eel (Anguilla anguilla) in the tidal area of a small estuary on the French Atlantic coast. Helgolander Meeresuntersuchungen, 40, 321-326. 
GASCUEL D., 1987. La civelle d'anguille dans l'estuaire de la Sèvre niortaise. Biologie, écologie et exploitation. Rapport. ENSA Rennes, Département Halieutique n² 4, 353 p.

GERMAIN L., 1927. La distribution géographique des anguilles. Bull. Soc. Océa. Fr., 35, 678-682.

ICES 2002. Report of the ICES/EIFAC working group on eels. ICES C.M. 2002/ACFM, 03.

JELLYMAN D.J., LAMBERT P.W., 2003. Factors affecting recruitment of glass eels into the Grey River, New Zealand. Journal of Fish Biology, 63, 1067-1079.

KING J.R., MAC FARLANE G.A., BEAMISH R.J., 2001. Incorporating the dynamics of marine systems into the stock assessment and management of sablefish. Progress in Oceanography, 49, 619-639.

KIRK R.S., 2003. The impact of Anguillicola crassus on European eels. Fisheries Management and Ecology, 10, 385-394.

LAFFAILLE P., FEUNTEUN E., ACOU A., LEFEUVRE J.-C., 2000. Role of European eel (Anguilla anguilla L.) in the transfer of organic matter between marine and freshwater systems. Verhandlungen Internationale Vereinigung für Limnologie, 27, 616-619.

LAFFAILLE P., ACOU A., GUILLOUËT J., 2003a. Evolution de la population d'anguille en 8 ans de suivi sur le Frémur, petit fleuve côtier de Bretagne nord. Colloque «les poissons migrateurs, un patrimoine régional ». Rennes, le 16 octobre 2003.

LAFFAILLE P., FEUNTEUN E., BAISEZ A., ROBINET T., ACOU A., LEGAULT A., LEK S., 2003b. Spatial organisation of European eel (Anguilla anguilla L.) in a small catchment. Ecology of Freshwater Fish, 12, 254-264.

LAFFAILLE P., BAISEZ A., RIGAUD C., FEUNTEUN E., 2004a. Habitat preferences of different European eel size classes in a reclaimed marsh: a contribution to species and ecosystem conservation. Wetlands, 24, 642-651.

LAFFAILLE P., LASNE E., CANARD A., 2004b. Mise en place d'une méthodologie d'analyse de la population d'anguilles sédentaires en relation avec les habitats disponibles à l'échelle du bassin versant Loire. Bilan de la première année (2004) et perspectives pour 2005 et 2006. Rapport Univerisité Rennes 1, Diren des Pays de la Loire, Région des Pays de la Loire, Agence de l'Eau Loire Bretagne, Tableau de Bord Anguilla Loire, $21 \mathrm{p}$.

LAFFAILLE P., BAISEZ A., LASNE E., 2004c. Freshwater Habitat of European eel. Ices Annual Conference, Vigo. ICES CM 2004/S: 02.

LAFFAILLE P., ACOU A., GUILLOUËT J., LEGAULT A., 2005a. Temporal changes in European eel, Anguilla anguilla, stocks in a small catchment after installation of fish passes. Fisheries Management and Ecology, 12, 123-129.

LAFFAILLE P., BRIAND C., FATIN D., LAFAGE D., LASNE E., 2005b. Point sampling the abundance of European eel (Anguilla anguilla) in freshwater areas. Archiv für Hydrobiologie, 162, 91-98.

LAMBERT P., FEUNTEUN E., 1998. Compte-rendu du Groupe Anguille du GRISAM (Paimpont, 1998), $45 \mathrm{p}$.

LEAUTE J.-P., CAILL-MILLY N., 2003. Caractéristiques des petites pêches côtières et estuariennes de la Côte atlantique du Sud de l'Europe. Synthèse du Programme Pecosude. Ifremer, Bilans et prospectives, $66 \mathrm{p}$.

LEFEBVRE F., SERGENT E., ACOU A., LECOMTE-FINIGER R., CRIVELLI A.J., 2003. Recruitment of glass eels (Anguilla anguilla) on the French Mediterranean coast: a comparative analysis of biometric and pigmentation characteristics during the 1974-75 and 2000-2001 sampling seasons. Bull. Fr. Pêche Piscic., 368, 85-96. 
LEFEUVRE J. -C., LAFFAILLE P., FEUNTEUN E., BOUCHARD V., RADUREAU A., 2003. Biodiversity in salt marshes: from the patrimonial value to the ecosystem functioning. The case study of the Mont Saint-Michel bay. Comptes Rendus Biologies, 326,125-131.

LEGAULT A., 1987. L'anguille dans le bassin de la Sèvre Niortaise. Biologie, Ecologie, Exploitation. Publication Halieutique ENSAR, 6, 305 p.

LEGAULT A., 1988. Le franchissement des barrages par l'escalade de l'anguille. Etude de la Sèvre Niortaise. Bull. Fr. Pêche Piscic., 308, 1-10.

LEGAULT A., 1990. Gestion des barrages estuariens et migration d'anguilles. Int. Revue Ges. Hydrobiol., 75, 819-825.

LEGAULT A., 1994. Étude préliminaire du recrutement fluvial de l'anguille. Bull. Fr. Pêche Piscic., 335, 33-42.

LOBON-CERVIA J., 1999. The decline of eel Anguilla anguilla (L.) in a river catchment of northern Spain 1986-1997. Further evidence for a critical status of eel on Iberian waters. Archiv für Hydrobiologie, 144, 245-253.

MAGALHAES M.F., BATALHA D.C., COLLARES-PEREIRA M.J., 2002. Gradients in stream fish assemblages across a Mediterranean landscape: contributions of environmental factors and spatial structure. Freshwater Biology, 47, 1015-1031.

MASSE J., RIGAUD C., 1998. Anguille et marais littoraux atlantiques. Collection IfremerActes de colloque $\mathrm{n}^{\circ} 19$, «Marais maritimes et aquaculture. Exploitation durable des zones humides littorales ", 141-153.

MORIARTY C., DEKKER W., 1997. Management of European eel fisheries. Fishery Bulletin, $15,1-110$.

OBERDORFF T., PONT D., HUGUENY B., BELLIARD J., BERREBI dit THOMAS R., PORCHER J.P., 2001. Adaptation et validation d'un indice poisson (FBI) pour l'évaluation de la qualité biologique des cours d'eau français. Bull. Fr. Pêche Piscic., 365/366, 405-433.

OUEST GRANDS MIGRATEURS, 16 Décembre 2003. Les poissons migrateurs Un patrimoine régional. Synthèses des débats et présentations, 73 pages.

RIGAUD C., 1997. Compte-rendu du Groupe Anguille du Grisam (Le Teich, 1997), 52 p.

ROBINET T., FEUNTEUN E., 2002. Sublethal effects of exposure to chemical compounds: a cause for the decline in Atlantic eels? Ecotoxicology, 11, 265-277.

RUSSEL I.C., POTTER E.C.E., 2003. Implications of the precautionary approach fort he management of the European eel, Anguilla anguilla. Fisheries Management and Ecology, 10, 395-401.

SCHMIDT J., 1922. The breeding place of the eels. Phil. Trans. R. Soc., 211, 179-208.

SIMBERLOFF D., 1998. Flagships, umbrellas, and keystones: is single-species management passé in the lansdscape area? Biological Conservation, 83, 247-257.

STEINBACH P., 2003. La libre circulation : conditions de colonisation du bassin versant et état réglementaire. Secondes Rencontres Scientifiques «L'anguille en Loire ». 7 mars 2003, Angers.

STEINBACH P., 2004. Expertise de la franchissabilité des ouvrages hydrauliques transversaux par l'anguille. Note méthodologique. Conseil Supérieur de la Pêche, $11 \mathrm{p}$.

WOILLEZ M., ROCHARD E., 2003. Tableau de bord, un outil pour le suivi des poisons migrateurs: concepts et méthode pour le choix des indicateurs, exemple de la population de grande alose du basin versant Gironde-Garonne-Dordogne. Ingénieries, 35, 43-59. 